\title{
Massive Dirac Fermion on the Surface of a magnetically doped Topological Insulator
}

\author{
Y. L. Chen ${ }^{1,2,3}$, J.-H. Chu ${ }^{1,2}$, J. G. Analytis ${ }^{1,2}$, Z. K. Liu ${ }^{1,2}$, K. Igarashi ${ }^{4}$, H.-H. Kuo ${ }^{1,2}$, X. L. Qi ${ }^{1,2}$, \\ S. K. Mo ${ }^{3}$, R. G. Moore ${ }^{1}$, D. H. Lu ${ }^{1}$, M. Hashimoto ${ }^{2,3}$, T. Sasagawa ${ }^{4}$, S. C. Zhang ${ }^{1,2}$, I. R. Fisher ${ }^{1,2}$, \\ Z. Hussain ${ }^{3}$ and Z. X. Shen ${ }^{1,2}$ \\ 1. Stanford Institute for Materials and Energy Sciences, SLAC National Accelerator Laboratory, \\ 2575 Sand Hill Road, Menlo Park, California 94025 \\ 2. Geballe Laboratory for Advanced Materials, Departments of Physics and Applied Physics, \\ Stanford University, Stanford, California 94305. \\ ${ }^{3}$ Advanced Light Source, Lawrence Berkeley National Laboratory \\ Berkeley California, 94720, USA. \\ ${ }^{4}$ Materials and Structures Laboratory, Tokyo Institute of Technology \\ Kanagawa, 226-8503, Japan
}

Topological insulators are characterized by a massless Dirac surface state and a bulk energy gap. An insulating massive Dirac fermion state is predicted to occur if the breaking of the time reversal symmetry opens an energy gap at the Dirac point, provided that the Fermi-energy resides inside both the surface and bulk gaps. By introducing magnetic dopants into the three dimensional topological insulator $\mathrm{Bi}_{2} \mathrm{Se}_{3}$ to break the time reversal symmetry, we observed the formation of a massive Dirac fermion on the surface; simultaneous magnetic and charge doping furthermore positioned the Fermi-energy inside the Dirac gap. The insulating massive Dirac Fermion state thus obtained may provide a tool for studying a range of topological phenomena relevant to both condensed matter and particle physics. 
Topological insulators are a new state of matter that may serve as a platform for investigating both fundamental physics and technological applications, such as spintronics and quantum information processing. Since the discovery in HgTe quantum wells $(1,2)$, topological insulators have been a very active research area (3-11). Recently, a class of three dimensional topological insulators $\mathrm{Bi}_{2} \mathrm{Te}_{3}, \mathrm{Bi}_{2} \mathrm{Se}_{3}$ and $\mathrm{Sb}_{2} \mathrm{Te}_{3}$ were identified (12-14) with the surface state consisting of a single Dirac cone. The conducting surface states of topological insulators are immune to localization as long as the disorder potential does not violate time reversal symmetry (TRS) $(4,5,9)$, and the only way to break this robust surface metallicity is to break the TRS by introducing magnetic order (5). In the bulk, a topological insulator doped with magnetic impurities can have a long range magnetic order both in the metallic (15-16) and insulating (17) phases; on the surface, such a long range magnetic order can also be formed independent of the bulk magnetic ordering, as the Ruderman-Kittel-Kasuya-Yosida (RKKY) interaction induced by the Dirac fermions is generally ferromagnetic when Fermi-energy $\left(E_{F}\right)$ is close to the Dirac point (18). Both effects can lead to the breaking of TRS, resulting in a gap opening at the Dirac point that makes the surface Dirac fermion massive. Furthermore, if $E_{F}$ can be tuned into this surface state gap, an insulating massive Dirac fermion state is formed; this state may support many striking topological phenomena, such as the image magnetic monopole induced by a point charge $(19,20)$, the half quantum Hall effect on the surface with a Hall conductance of $\mathrm{e}^{2} / 2 \mathrm{~h}$, and a topological contribution to the Faraday and Kerr effects (5). In addition, this state is a concrete realization of the " $\theta$ vacuum" state of axion physics in a condensed matter system (5), and thus has implications for particle physics and cosmology (21). Finally, a tunable energy gap at the surface Dirac point provides a means to control the surface electric transport, which is of great importance for applications.

The insulating massive Dirac fermion state is challenging to realize as there are two critical requirements that must be simultaneously satisfied: First, a gap opens at the Dirac point of 
the topological surface state (due to the braking of TRS); second, $E_{F}$ of the system must reside inside both the surface and bulk gap. We report the realization of this state with simultaneous fulfilment of both requirements in the topological insulator $\mathrm{Bi}_{2} \mathrm{Se}_{3}$ by introducing an exact amount of magnetic dopants to break the TRS and precisely control the $E_{F}$ position.

We performed angle-resolved photoemission spectroscopy (ARPES) to investigate the electronic structures of intrinsic, non-magnetically and magnetically doped $\mathrm{Bi}_{2} \mathrm{Se}_{3}$ (22). Figure 1 illustrates the measured band structure of undoped $\mathrm{Bi}_{2} \mathrm{Se}_{3}$. Similar to $\mathrm{Bi}_{2} \mathrm{Te}_{3}(14)$, besides the Fermi-surface (FS) pocket from the surface state band (SSB), there is also a FS pocket from the bulk conduction band (BCB) (Fig. 1A-D) due to the Se deficiencies and the Bi-Se inter-site defects. The bottom of the BCB is located at $190 \mathrm{meV}$ above the Dirac point (Fig. 1A,C), indicating a direct bulk gap (22). The in-gap Dirac point makes $\mathrm{Bi}_{2} \mathrm{Se}_{3}$ a better candidate for realizing the insulating massive Dirac fermion state than $\mathrm{Bi}_{2} \mathrm{Te}_{3}$, in which the Dirac point is below the top of the bulk valence band (BVB) (14) thus demanding a much larger surface energy gap for $E_{F}$ to reside inside both the surface and bulk gaps. The cross section plot of the band structure (Fig.1B) shows how the SSB evolves from the Dirac point to a hexagonal shape at $E_{F}$. Different from the $\mathrm{Bi}_{2} \mathrm{Te}_{3}$ band structure where the SSB FS starts being warped at energies close to the BCB minimum (14) and becomes a concave hexagram, the SSB FS of $\mathrm{Bi}_{2} \mathrm{Se}_{3}$ remains convex hexagonal even in the presence of the BCB. This difference will result in different observations in other experiments, such as scanning tunneling microscopy/spectroscopy (STM/STS), where the surface quasi-particle interference around defects can be suppressed in $\mathrm{Bi}_{2} \mathrm{Se}_{3}$, unlike in $\mathrm{Bi}_{2} \mathrm{Te}_{3}$ where the concave SSB FS shape favors such scattering along specific directions (23-27).

The surface nature of the hexagonal SSB FS is confirmed by the photon energydependent ARPES study (Fig.1D), where its non-varying shape with different excitation photon 
energies indicates its two dimensional nature. By contrast, the shape and the existence of the inner BCB FS pocket changes markedly due to its three dimensional nature with strong $k_{z}$ dispersion.

In the presence of TRS, the SSB of $\mathrm{Bi}_{2} \mathrm{Se}_{3}$ is degenerate at the Dirac point which connects the upper and lower surface Dirac cone (Fig. 2B) even if the system is perturbed by nonmagnetic dopants (Fig. 2A). This is confirmed by the ARPES measurements (Fig. 2C,D), where the band structures of an intrinsic and a nominally $10 \% \mathrm{Tl}$ doped samples are shown, respectively. In both cases, the continuity at the Dirac point is indicated by the strong spectral intensity (left sub-panels) and the single peak structure of the energy distribution curve (EDC) at the Dirac point (right sub-panels). In Fig. 2D, the charge doping effect of $\mathrm{Tl}$ is clearly shown by the dramatic shift of $E_{F}$ into the bulk gap $\left(E_{F}-E_{D}=160 \mathrm{meV}\right)$. Nonetheless, the topology of the SSB remains the same with a continuous Dirac point (22).

The TRS protection of the Dirac point can be lifted by magnetic dopants (Fig. 2E), resulting in a gap that separates the upper and lower branches of the Dirac cone (Fig. 2F). This is illustrated in the band structure (Fig. 2G,H) of two Fe-doped samples. Unlike non-magnetically doped samples, for both Fe-doped samples, the SSB dispersion at the Dirac point is broken, as indicated by the suppressed intensity regions in the spectral density plots (left sub-panels) and the twin-peak structure around the Dirac point in the EDC plots (right sub-panels). The data have sufficient $k$-space sampling density to reveal the qualitative difference between the non-magnetic and magnetic dopants: one always finds a single peak structure in as-grown and non-magnetically doped samples, while the twin-peak structure is only present in magnetically doped samples (22). By fitting the twin peak structure with two Lorentzian peaks (insets in EDC plots of Fig. 2G,H), the gap size can be acquired, showing a larger value $(\sim 50 \mathrm{meV})$ in Fig. $2 \mathrm{H}$ than that $(\sim 44 \mathrm{meV})$ in 
Fig. 2G. This trend (22) is consistent with the increase of the magnetic moment upon increasing the magnetic dopants concentration.

The SSB gap formation at the Dirac point with broken TRS is the first step to realize the insulating massive Dirac fermion state, and the second step is to tune the $E_{F}$ into this gap. In the Fe-doped $\mathrm{Bi}_{2} \mathrm{Se}_{3}$, however, $E_{F}$ was found to always reside above the Dirac point (similar to undoped $\mathrm{Bi}_{2} \mathrm{Se}_{3}$ ), making the material n-type (Fig. 2G,H). To remove these excess n-type carriers while maintaining the magnetic doping effect, we change the dopant from Fe to $\mathrm{Mn}$, another magnetic material with one less valence electron than Fe. Indeed, Mn dopants not only introduce magnetic moments into the system, but also naturally p-dope the samples. The measurements on an optimally doped sample (22) show $E_{F}$ residing right inside the SSB gap (Fig. 3B). By comparing the leading edge of the EDC at $\Gamma$ point to $E_{F}$ (Fig. 3C, also shown is an Au reference spectrum), we found a $7 \mathrm{meV}$ difference, indicating a SSB Dirac gap of at least 7meV (Fig. 3A). Such a gap suggests a ferromagnetic order of the Mn dopants on the surface, which can be induced by the ferromagnetic spin-spin interaction mediated by the surface states (18). This optimally doped sample thus fully realizes the insulating massive Dirac fermion state and provides a model system for studying exciting topological phenomena.

To maintain this insulating massive Dirac fermion state at higher temperatures, further increase of the Dirac gap (while keeping $E_{F}$ inside it) is needed. However, because of the holedoping effect of Mn dopants, one cannot simply increase the Mn concentration in $\left(\mathrm{Bi}_{1-\delta} \mathrm{Mn}_{\delta}\right)_{2} \mathrm{Se}_{3}$ to acquire a larger Dirac gap, as the system will become p-type before the gap magnitude increases appreciably (22). However, as we found it was possible to introduce much Fe dopants into $\mathrm{Bi}_{2} \mathrm{Se}_{3}$ to increase the gap size without substantially altering the $E_{F}$ position comparing to the 
undoped $\mathrm{Bi}_{2} \mathrm{Se}_{3}$, if we can further adjust $E_{F}$ into the gap by introducing additional p-type dopants, a larger gap insulating massive Dirac fermion state can be achieved.

Figure 4 demonstrates the full range of $E_{F}$ tuning by introducing such p-type doping, with three doping regions and the topological transport point (where $\mathrm{E}_{\mathrm{F}}$ coincides with the Dirac point) shown in Fig. 4A. By either surface [Fig. 4B and (22)] or bulk doping (Fig. 4C-F), we were able to tune the $E_{F}$ to any of the regions defined in Fig. 4A. The ability to convert the original n-type sample to p-type by surface doping (Fig. 4B, region iii) is critical for applications requiring both types of carrier dopings or p-n junctions. On the other hand, the full range bulk doping (Fig. 4C-F) has the advantages over the surface doping in bulk applications. 


\section{References and notes:}

1. A. Bernevig, T. L. Hughes, S.-C. Zhang, Quantum spin Hall effect and topological phase transition in HgTe quantum wells, Science 314, 1757 (2006)

2. M. König et al., Quantum spin Hall insulator state in HgTe quantum wells, Science 318, 766 (2007)

3. X. L. Qi, S. C. Zhang, The quantum spin Hall effect and topological insulators, Phys. Today 63, 33 (2010)

4. L. Fu, C. L. Kane, E. J. Mele Topological insulators in three dimensions, Phys. Rev. Lett. 98, 106803 (2007)

5. X. L. Qi, T. L. Hughes, S. C. Zhang, Topological field theory of time-reversal invariant insulators, Phys. Rev. B 78, 195424 (2008)

6. A. P. Schnyder, S. Ryu, A. Furusaki, A. W. Ludwig, Classification of topological insulators and superconductors in three spatial dimensions, Phys. Rev. B 78, 195125 (2008).

7. L. Fu, C. L. Kane, Probing neutral Majorana fermion edge modes with charge transport, Phys. Rev. Lett. 102, 216403 (2009).

8. A. R. Akhmerov, J. Nilsson, C. W. J. Beenakker, Electrically detected interferometry of Majorana fermions in a topological insulator, Phys. Rev. Lett 102, 216404 (2009).

9. J. E. Moore, L. Balents, Topological invariants of time-reversal-invariant band structures, Phys. Rev. B 75, 121306(R) (2007).

10. R. Roy, $Z_{2}$ classification of quantum spin Hall systems: An approach using time-reversal invariance, Phys. Rev. B 79, 195321 (2009)

11. B. Seradjeh, J. E. Moore, M. Franz, Exciton condensation and charge fractionalization in a topological insulator film, Phys. Rev. Lett, 103, 066402 (2009)

12. H. Zhang, et al., Topological insulators in $\mathrm{Bi}_{2} \mathrm{Se}_{3}, \mathrm{Bi}_{2} \mathrm{Te}_{3}$ and $\mathrm{Sb}_{2} \mathrm{Te}_{3}$ with a single Dirac cone on the surface, Nature Phys. 5, 438 (2009). 
13. Y. Xia, et al., Observation of a large-gap topological-insulator class with a single Dirac cone on the surface, Nature Phys. 5, 398 (2009).

14. Y. L. Chen, et al., Experimental realization of a three-dimensional topological insulator, $\mathrm{Bi}_{2} \mathrm{Te}_{3}$, Science 325178 (2009)

15. J. Choi, et. al., Magnetic properties of $\mathrm{Mn}$-doped $\mathrm{Bi}_{2} \mathrm{Te}_{3}$ and $\mathrm{Sb}_{2} \mathrm{Te}_{3}$, Phys. Stat. Sol. (b) 2411541 (2004)

16. Y. S. Hor et. al., Development of ferromagnetism in the doped topological insulator $\mathrm{Bi}_{2-\mathrm{x}} \mathrm{Mn}_{\mathrm{x}} \mathrm{Te}_{3}$, Phys. Rev. B 81195203 (2010).

17. R. Yu, et al., Quantized Anomalous Hall Effect in Magnetic Topological Insulators, http://arxiv.org/abs/1002.0946

18. Q. Liu, C. X. Liu, C. K. Xu, X. L. Qi, S. C. Zhang, Magnetic Impurities on the Surface of a Topological Insulator, Phys. Rev. Lett. 102156603 (2009)

19. X. L. Qi, R. D. Li, J. Zang, S. C. Zhang, Inducing a magnetic monopole with topological surface states , Science 323, 1184 (2009)

20. J. Zang, N. Nagaosa, Monopole Current and Unconventional Hall Response on Topological Insulator, http://arxiv.org/abs/1001.1578

21. F. Wilzcek, A theoretical physicist examines exotic particles lurking in new materials. Nature, 458, 129 (2009)

22. Materials, methods and addition information are available as supporting online materials on Science Online.

23. L. Fu, Hexagonal warping effects in the surface states of the topological insulator $\mathrm{Bi}_{2} \mathrm{Te}_{3}$, Phys. Rev. Lett. 103266801 (2009)

24. X. Zhou, C. Fang, W. F. Tsai, J. P. Hu, Theory of quasiparticle scattering in a twodimensional system of helical Dirac fermions: Surface band structure of a threedimensional topological insulator, Phys. Rev. B 80, 245317 (2009) 
25. W. C. Lee, C. Wu, D. P. Arovas, S. C. Zhang, Quasiparticle interference on the surface of the topological insulator $\mathrm{Bi}_{2} \mathrm{Te}_{3}$, Phys. Rev. B 80, 245439 (2009)

26. Z. Alpichshev et al., STM imaging of electronic waves on the surface of Bi2Te3: topologically protected surface states and hexagonal warping effects, Phys. Rev. Lett. 104 $016401(2010)$

27. T. Zhang, et al., Experimental demonstration of the topological surface states protected by the time-reversal symmetry, http://arxiv.org/abs/0908.4136

28. This work is supported by the Department of Energy, Office of Basic Energy Science under contract DE-AC02-76SF00515. 
Fig. 1. Electronic band structure of undoped $\mathrm{Bi}_{2} \mathrm{Se}_{3}$ measured by ARPES. (A) The bulk conduction band (BCB), bulk valence band (BVB), surface state band (SSB) and the Dirac point are indicated, along with the Fermi-energy $\left(E_{F}\right)$, bottom of the BCB $\left(E_{B}\right)$ and the Dirac point $\left(E_{D}\right)$. (B) Constant-energy contours of the band structure show the SSB evolution from the Dirac point to a hexagonal shape (green dashed lines). (C) Band structure along the $\mathrm{K}-\Gamma-\mathrm{K}$ direction. The BCB bottom is about $190 \mathrm{meV}$ above $E_{D}$ and $150 \mathrm{meV}$ below $E_{F}$. (D) Photon energy dependent FS maps (symmetrized according to the crystal symmetry). Blue dashed lines around the BCB FS pocket indicate their different shapes. 
Fig. 2. (A, B) A non-magnetically doped topological insulator with a Dirac point connecting the upper and lower Dirac cones as in the undoped case. (C) Band structure along the $\mathrm{K}-\Gamma-\mathrm{K}$ direction of undoped $\mathrm{Bi}_{2} \mathrm{Se}_{3}$. Left and right sub-panels show the ARPES spectral intensity plot and a stacking plot of the energy distribution curves, respectively. Red curve in the right subpanel indicates the EDC at $\Gamma$ point. Inset: EDC at the $\Gamma$ point (red), fitted with a Lorentzian peak (green) on the Shirley background (black); the total fitting function is shown in blue. The same convention is used in panels (D, G, H). (D) Band structure for a Tl-doped sample $\left(\mathrm{Bi}_{0.9} \mathrm{Tl}_{0.1}\right)_{2} \mathrm{Se}_{3}$. The Dirac point remains continuous. (E, F) A magnetically doped topological insulator with a broken Dirac point and a gap separating the upper and lower Dirac cones. (G, H) Band structure of two Fe-doped samples (from two growth batches with melt composition $\left(\mathrm{Bi}_{0.88} \mathrm{Fe}_{0.12}\right)_{2} \mathrm{Se}_{3.7}$ and ( $\left.\mathrm{Bi}_{0.84} \mathrm{Fe}_{0.16}\right)_{2} \mathrm{Se}_{3.7}$, respectively). At the Dirac point, the reduced spectral intensity (left sub-panels) and the twin-peak structure in the EDCs (right sub-panels) indicate a gap formation. 
Fig. 3. Realization of the insulating massive Dirac fermion state by simultaneous magnetic and charge doping. (A) Gap formation at the Dirac point (caused by magnetic impurities on the surface) and the in-gap $E_{F}$ position. The occupied (unoccupied) Dirac cone is shown in blue (gray); $\Delta$ is the energy difference between the top of the occupied Dirac cone and $E_{F}$. (B) ARPES spectra intensity plot of the band structure along the $\mathrm{K}-\Gamma-\mathrm{K}$ direction of a Mn-doped sample $\left(\mathrm{Bi}_{0.99} \mathrm{Mn}_{0.01}\right)_{2} \mathrm{Se}_{3}$ showing the $E_{F}$ inside the surface Dirac gap. Inset: close-up of the dispersion in the vicinity of $E_{F}$, indicating a gap between the leading edge of the SSB and $E_{F}$. Vertical white dashed line shows the location of the EDC plotted in (C). (C) Comparison between the $\Gamma$ point EDC (blue) and $E_{F}$ shows a $7 \mathrm{meV}$ leading edge gap (EDC on the full energy scale is plotted in the inset). A reference EDC from a polycrystalline Au sample whose leading edge, as expected, coincides with $E_{F}$ is shown in red. 
Fig. 4. Full range control of $E_{F}$ position by surface or bulk doping. (A) Different carrier type regions: n-type (region I), bulk insulating (region II), Dirac transport and p-type (region III) regions determined by the $E_{F}$ position. (B) Evolution of the band structure (along the $\mathrm{M}-\Gamma-\mathrm{M}$ direction) by photon-assisted surface doping with $\mathrm{O}_{2}(22)$. The blue dashed line traces the up-shift of the Dirac point with the $\mathrm{O}_{2}$ doping. Green dashed lines indicate the dosages that separate the three doping regions shown in (A): At $0.95 \mathrm{~L} \mathrm{O}_{2}$ dosage, the BCB bottom reaches $E_{F}$; at $3.6 \mathrm{~L}$, the Dirac point reaches $E_{F}$; and beyond 3.6L, the Dirac point is above $E_{F}$. Bulk doping: the FS and the band structure of a $(\mathbf{C})$ Nominally undoped $\mathrm{Bi}_{2} \mathrm{Se}_{3}$, showing the coexistence of BCB and SSB FS pockets. (D) Se rich sample (melt composition $\mathrm{Bi}_{1.7} \mathrm{Se}_{3.3}$ ) with only an SSB FS, and $E_{F}$ residing inside the bulk gap $\left(E_{F}-E_{D}=145 \mathrm{meV}\right)$. (E) Mg-doped $\left(\left(\mathrm{Bi}_{0.999} \mathrm{Mg}_{0.001}\right)_{2} \mathrm{Se}_{3}\right)$ sample with a point-like FS and $E_{F}$ precisely at the Dirac point. (F) More richly Mg-doped sample ( $\left.\left(\mathrm{Bi}_{0.998} \mathrm{Mg}_{0.002}\right)_{2} \mathrm{Se}_{3}\right)$ driven into p-type, with a p-type FS and the Dirac point above $E_{F}$. 